\title{
Concrete Hydration Model Characterization Using Evolutionary Optimization
}

\author{
Qianchen Sun \\ qs217@cam.ac.uk \\ Department of Engineering, University of Cambridge, Cambridge, UK \\ Mohammed Z.E.B. Elshafie \\ melshafie@qu.edu.qa \\ Department of Civil and Architectural Engineering, Qatar University, Doha, Qatar \\ Yi Rui \\ yr228@cam.ac.uk \\ Department of Engineering, University of Cambridge, Cambridge, UK
}

\begin{abstract}
Pile thermal integrity assessment by means of temperature measurement has received increasing attention in recent years. The thermal integrity testing method measures temperature changes during the concrete curing process; using an appropriate concrete hydration model together with tracking temperature development during the curing process, defects within piles could be detected. However, the implementation of thermal integrity testing in practice faces, potentially, many uncertainties including undocumented concrete mixes, lack of knowledge of ground thermal properties, uncertain boundary conditions for pile, etc... These uncertainties increase the complexity of determining appropriate parameters for the hydration model, which directly affects the defect detection capacity of the method. This paper presents an inverse approach using differential evolution (DE) algorithms to determine the concrete hydration model. With this approach, the finite element (FE) analysis is integrated into the DE algorithm to generate approximate solutions that match a controlled dataset instead of approximating the concrete hydration parameters with limited prior knowledge as currently used in practice. Firstly, a field test temperature dataset with a well-defined boundary condition is selected. The temperature development corresponding to the selected dataset is then numerically simulated using an uncalibrated general hydration model. Finally, the hydration model parameters are determined using DE algorithms based on the measured and simulated temperature development as inputs. A field case study is presented in the end of this paper. The results indicate that the proposed inverse approach using DE algorithms can be used effectively in thermal integrity testing.
\end{abstract}

Keywords: Differential evolution; Thermal integrity test; Pile anomaly detection; Finite element modelling; Structural health monitoring

\section{INTRODUCTION}

Pile foundations provide support for structures by transferring the load into deeper layers of stiff soil or rock. Although pile foundations have been widely used in construction, there are still challenges about pile inspection and quality assurance. Due to practical construction issues and/or lack of knowledge of ground conditions, anomalies such as voids, soil inclusion, necking and poor concrete quality can exist inside pile 
concrete body. These integrity problems could severely affect the load bearing capacity of concrete piles. Whereas, it is not possible to inspect the pile visually during or after installation. Therefore, pile quality control and quality assurance has been an important but challenging task.

A new thermal integrity testing method has been proposed recently for pile defect detection. The fundamental mechanism is that early age concrete release heat during the hydration process, temperature signatures within the concrete body will change if defects exist. Distributed temperature sensors are used for temperature measurement; the method then employs concrete hydration model and finite element analysis to predict temperature development, which is then compared with field test temperature data. The comparison between thermal integrity testing data and numerical data can provide further insights to identify the nature and geometry of anomalies. Details of this method can be found in Rui et al. (2017) and (Sun \& Elshafie, 2019).

The capacity of the thermal integrity method to detect defect depends on the accuracy of the hydration model. The general practice is to use a general hydration heat generation graph based on limited knowledge of concrete mixes and ground conditions to predict the theoretical concrete temperature development. In this paper, an inverse approach is proposed which uses measured temperature data to analyze the actual hydration development. The finite element (FE) analysis is integrated into a differential evolution (DE) algorithm to generate approximate solutions that match a controlled dataset.

In this paper, the fundamental mechanism of concrete hydration will be presented first, followed by the proposed evolutionary optimization for the hydration model. Then a field case study will be presented to demonstrate the proposed method. Conclusions will be drawn at the end of the paper.

\section{CONCRETE HYDRATION MODEL}

The concrete hydration reaction is a thermally activated and exothermic process. The amount of heat, which is liberated within the first few days of concrete casting, plays a crucial role in temperature development of early age concrete. Temperature abnormities can be a good indication of concrete anomalies.

An early classic hydration model is developed by (De Schutter \& Taerwe, 1995), based on a series of isothermal cement calorimetry tests and concrete adiabatic tests. In this model, the heat production rate $\dot{Q}$ is expressed as a function of temperature and degree of hydration:

$$
\dot{Q}(t)=q_{\max , 20} \cdot c \cdot\left[\sin \left(\alpha_{t} \pi\right)\right]^{a} \cdot e^{-b \alpha_{t}} \cdot e^{-\left[\frac{E}{R}\left(\frac{1}{T_{c}}-\frac{1}{T_{r}}\right)\right]}
$$

where $a, b$ and $c$ are the material constants controlling the distribution of hydration heat production; $\alpha_{t}$ is the degree of hydration, defined as the fraction of the heat of hydration that has been released $\left(Q_{\text {released }} / Q_{\text {total }}\right) ; E$ is the apparent activation energy, $R$ is the universal gas constant, $q_{\max , 20}$ is the is the maximum heat production rate at 20 ${ }^{\circ} \mathrm{C}, T_{c}$ is the temperature of concrete (K), and $T_{r}$ is the is the reference temperature (293 $\mathrm{K})$. This model has a simply mathematical expression and gives a relatively accurate 
hydration heat prediction, but it has limitation for simulating Type III cement. Another hydration model is shown below to tackle this issue.

Another hydration model is developed based on the equivalent age maturity theory and Arrhenius rate theory (Schindler, 2004; Schindler \& Folliard, 2005; Riding et al., 2006). The main advantages of this model are suitability of various cement types and better representation of temperature effect by applying equivalent age $\left(t_{\varepsilon}\right)$ (Malhotra \& Carino, 2003). The model is mathematically expressed as follows:

$$
\begin{aligned}
& t_{\varepsilon}=\sum_{0}^{t} \exp \left(\frac{E}{R}\left(\frac{1}{273+T_{r}}-\frac{1}{273+T_{c}}\right)\right) \cdot \Delta t \\
& \dot{Q}(t)=H_{u} \cdot\left(\frac{\tau}{t_{\varepsilon}}\right)^{\beta} \cdot \frac{\beta}{t_{\varepsilon}} \cdot \alpha_{u} \cdot \exp \left(-\left[\frac{\tau}{t_{\varepsilon}}\right]^{\beta}\right) \cdot \exp \left(\frac{E}{R}\left(\frac{1}{273+T_{r}}-\frac{1}{273+T_{c}}\right)\right)
\end{aligned}
$$

where $\tau$ = hydration time parameter, $\beta=$ hydration shape parameter, and $\alpha_{u}=$ ultimate degree of hydration, $H_{u}=$ total heat of hydration.

Using the hydration models above, the heat generation, transient heat flow and boundary conditions could be implemented using FE analysis through MATLAB. It would predict temperature profiles of early age concrete of different mixes and element geometries. However, the hydration model application in practice faces many uncertainties, including undocumented concrete mixes, lack of knowledge of ground thermal properties, uncertain boundary conditions for piles, etc... These uncertainties increase the complexity of determining appropriate hydration parameters, which directly affects the defect detection capacity of the method. The next section introduces an inverse approach using evolutionary algorithms to better estimate the concrete hydration model parameters.

\section{DIFFERENTIAL EVOLUTION}

An optimization algorithm known as differential evolution (DE) is adopted for this purpose. DE is an efficient optimization algorithm first introduced by (Storn \& Price, 1997) for global optimization over continuous parameter spaces. It is conceptually similar to other evolutionary algorithms such as genetic algorithms and is not prone to converging at local maxima. The $\mathrm{DE}$ algorithm has been effectively used in many recent engineering applications, such as geotechnical modelling (Uchida et al., 2016), damage detection (Jena et al., 2013; Liu \& Mao, 2016) and superstructure-foundation interaction (Leung et al., 2017).

\subsection{Randomize Initial Population}

In this process, NP initial random solutions (parameter sets) are first generated, where NP is the population size. The solutions are expressed in vectors form $\boldsymbol{\theta}_{i}$ as sown in Equation 4, known as target vector, which is evolved over $G$ generations to reach an optimal solution. Each target vector component has $D$-dimension element, where $D$ is 
the number of variables which equals to six corresponding to hydration parameters in this study $\left(a, b, c, E, q_{\max _{2 n}}, Q_{\text {total }}\right)$. Storn and Price (1997) suggested that a reasonable population size $N P$ should be between 5 to 10 times of the number of variables $D$.

$$
\boldsymbol{\theta}_{i, G}=\left\{\theta_{i, G}^{1}, \theta_{i, G}^{2}, \ldots, \theta_{i, G}^{D}\right\}, i=1,2, \ldots, N P
$$

In the concrete hydration problem, the search space of each variable is bounded by cement and concrete mix properties. The upper and lower bounds of these variables are

defined as $\boldsymbol{\theta}_{\min }=\left\{\theta_{\min }^{1}, \theta_{\min }^{2}, \ldots, \theta_{\min }^{D}\right\}$ and $\boldsymbol{\theta}_{\max }=\left\{\theta_{\max }^{1}, \theta_{\max }^{2}, \ldots, \theta_{\max }^{D}\right\}$ respectively. The maximum and minimum values can be found in (De Schutter \& Taerwe, $1995,1996)$. The initial value of $j$ th variable for $i$ th solution at generation zero ( $G=0$ ) can be expressed by the Equation 5, where rand stands for a uniformly distributed random number in the interval $(0,1)$ :

$$
\theta_{i, 0}^{j}=\theta_{\min }^{j}+\operatorname{rand} \cdot\left(\theta_{\max }^{j}-\theta_{\min }^{j}\right)
$$

\subsection{Mutation}

At each subsequent generation, mutant vectors $\boldsymbol{v}_{i}$ are formed by adding the amplified differential variation from two target vectors to a third one, expressed by the following equation:

$$
\boldsymbol{v}_{i, G+1}=\boldsymbol{\theta}_{r 1, G}+F \cdot\left(\boldsymbol{\theta}_{r 2, G}-\boldsymbol{\theta}_{r 3, G}\right)
$$

where $r 1, r 2, r 3$ are mutually different random indexes chosen from integer set $\{1,2,3, \ldots, N P\}$ and $F$ is a factor with range $[0,2]$ controlling the amplification of the differential variation. A larger $F$ enables the algorithm to search in a wider scope within the domain of each parameter, thus empowering it with a better exploration ability. In the optimization later stage, as the solutions converge, the population variation becomes smaller. Thus, a smaller $\mathrm{F}$ value empowers the algorithm with a better local exploration and amendatory ability. Figure 1 is a schematic representation of the mutation process for a simple two-dimensional objective function.

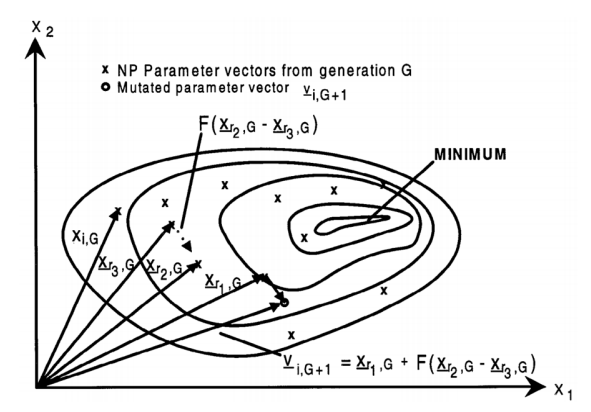

Figure 1: Illustration of operation process of mutation (Storn \& Price, 1997) 


\subsection{Crossover}

To increase the population diversity, "crossover" is introduced to determine the trial vectors $\boldsymbol{u}_{i}$ (i $\left.=1,2, \ldots, \mathrm{NP}\right)$. The operation is carried out by randomly exchanging components of mutation vectors $\boldsymbol{v}_{i, G+1}$ and the target vectors in the preceding generation $\boldsymbol{\theta}_{i, G}$, which produces the trail vectors $\boldsymbol{u}_{i, G+1}=\left\{u_{i, G+1}^{1}, u_{i, G+1}^{2}, \ldots, u_{i, G+1}^{D}\right\}$. The chosen for each component of $\boldsymbol{u}_{i}$ is formulated as follows:

$$
u_{i, G+1}^{j}= \begin{cases}v_{i, G+1}^{j} & \text { if }(\text { rand } \leq \mathrm{CR}) \text { or } j=j_{\text {rand }} \\ \theta_{i, G}^{j} & \text { otherwise. }\end{cases}
$$

where $\mathrm{CR}$ is the crossover constant within the range $[0,1]$, and $j_{\text {rand }}$ is a random integer from $[1, \mathrm{D}]$. Figure 2 illustrates an example crossover operation for 7-variable vectors.

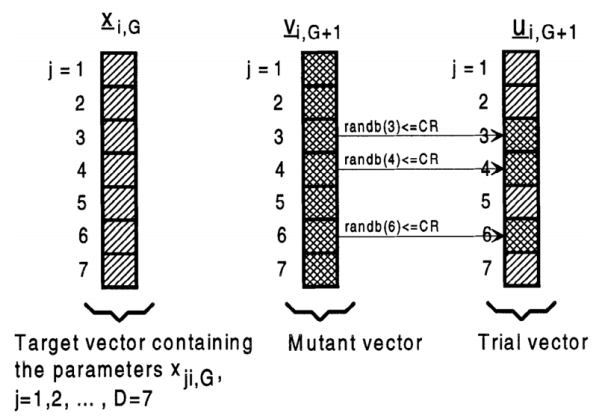

Figure 2: Illustration of operation process of crossover (Storn \& Price, 1997)

\subsection{Selection}

The fitness of target vector $\boldsymbol{\theta}_{i, G}$ and trail vector $\boldsymbol{u}_{i, G+1}$ are compared and evaluated through an objective function $f$. If $\boldsymbol{u}_{i, G+1}$ yields a smaller function value, then $\boldsymbol{\theta}_{i, G}$ $\boldsymbol{\theta}_{i, G}$ is replaced by $\boldsymbol{u}_{i, G+1}$; otherwise the original value $\boldsymbol{\theta}_{i, G}$ is retained in $(G+1)$ generation. The selection process is operated over the entire $N P$ population.

\subsection{Objective function}

Heat generation of early age concrete is controlled by hydration model. Variations of hydration model parameters make changes in concrete temperature prediction. In this study, the field test temperature development is simulated with a finite element (FE) program, which employs De Schutter's hydration model for predicting concrete curing heat. The objective function $f$ for determining a suitable hydration model is treated as an optimization problem. The optimal set of hydration parameters $\boldsymbol{\theta}^{*}$, as shown in Equation 8 , is obtained by minimizing the total difference between the field test temperature data $\left(T_{\text {test }}\right)$ and the FE model simulated temperature $(T(\boldsymbol{\theta}))$.

$$
\boldsymbol{\theta}^{*}=\arg \min _{\boldsymbol{\theta}} f(\boldsymbol{\theta})=\arg \min _{\boldsymbol{\theta}} \sum_{t}\left\|T(\boldsymbol{\theta})-T_{\text {test }}\right\|
$$




\section{FIELD CASE STUDY}

A field case study of thermal integrity testing has been conducted in London. This project involved the construction and monitoring of a test CFA pile. The test pile is used for validating the thermal integrity method and the proposed concrete hydration evolutionary optimization. The length of the pile was $20 \mathrm{~m}$ with a nominal diameter of $900 \mathrm{~mm}$ and a reinforcement cage diameter of $750 \mathrm{~mm}$. Three temperature cables were vertically attached to the reinforcement cage and measured the temperature at 15-minute intervals. Three one-meter long engineering inclusions were placed inside the reinforcement cage at depths of $3 \mathrm{~m}, 6 \mathrm{~m}$ and $9 \mathrm{~m}$. The inclusions were made of sandbags or containers with sand in order to simulate the defects such as loss of concrete cover and soil inclusions. More details on the test site and instrumentation used can be found in (Sun et al., 2019).

In this study, the measured temperature dataset between $14 \mathrm{~m}$ and $18 \mathrm{~m}$ depth was selected as the baseline temperature for calibrating the hydration model. No engineering defect was placed at this depth and the temperature profiles were consistently relatively stable. The average temperature data of three cables along $14 \mathrm{~m}-18 \mathrm{~m}$ were selected as the baseline temperature $\left(T_{\text {test }}\right)$ for optimization.

For efficient optimization of the hydration parameters, DE algorithms were employed for characterizing the hydration model. The concrete hydration heat is defined by a total of six parameters, grouped as a vector $\boldsymbol{\theta}=\left\{a, b, c, E, q_{\max _{2 \mathrm{n}}}, Q_{\text {total }}\right\}$. Firstly, 30 candidate solutions, known as 'target vectors', were first generated randomly in the optimization process as the initial generation population $G_{0}$. At each generation, 'mutant vectors' were formed by linear interpolation and multiplication of target vectors randomly selected from the population. A new set of 'trial vectors' was then generated by random mixture of the mutant vectors and the trial vectors in the previous generation.

Fitness of target vectors and trial vectors from the old and the new generations were compared through an objective function, which evaluates the discrepancies between FE model simulated temperature data and the temperature profiles obtained from field test results in 14-18m depth. This discrepancy was defined as an objective function $f(\boldsymbol{\theta})$. The fitter solutions remain in the new population, while the weaker ones are discarded. The procedures above were continuously iterated until a global optimum solution was achieved.
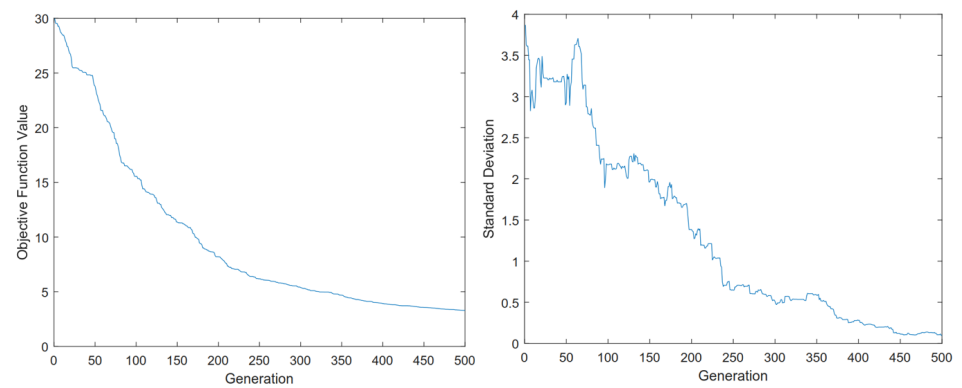

Figure 3: (a) Objective function value evolution (b) Standard deviation evolution 
Figure 3 (a) and (b) show the changes of average objective function value $f(\boldsymbol{\theta})$ and changes of the standard deviation of objective function values of each iteration. The function value rapidly reduced in the first 350 generations, and then became steady at a function value of around 5. The standard deviation of candidate solutions in each generation also continuously decreased and then maintained around 0.1 after $400^{\text {th }}$ generation. At $500^{\text {th }}$ generation, the global optimum was achieved, and the optimized hydration model parameters were obtained as shown in Table 1. The temperature prediction from the calibrated hydration parameters and the baseline temperature can be found in Figure 4. The temperature in the figure represent the temperature change compared to the initial baseline temperature $23^{\circ} \mathrm{C}$. It shows a good agreement with field test average temperature between $14-18 \mathrm{~m}$ depth.

Table 1 Optimized hydration model parameters

\begin{tabular}{|c|c|c|c|c|c|c|}
\hline Parameters & $\boldsymbol{a}$ & $\boldsymbol{b}$ & $\boldsymbol{c}$ & $\begin{array}{c}\boldsymbol{E} \\
\mathrm{kJ} / \mathrm{mol}\end{array}$ & $\begin{array}{c}\boldsymbol{q}_{\max 20} \\
\mathrm{I} / \mathrm{gh}\end{array}$ & $\begin{array}{c}Q_{\text {total }} \\
\mathrm{I} / \mathrm{gh}\end{array}$ \\
\hline Cement & 0.787 & 3.3 & 3.0 & 28.0 & 9.91 & 161 \\
\hline
\end{tabular}

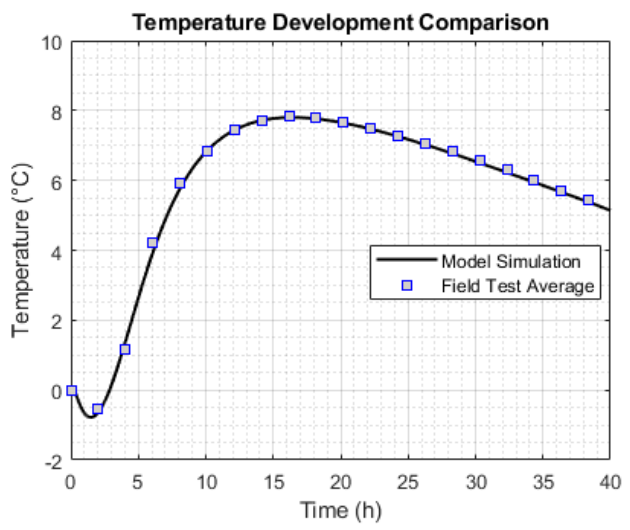

Figure 4: Temperature comparison between optimized model simulation and field test

\section{CONCLUSION}

The key element in thermal integrity testing is to determine an accurate concrete hydration model which controls temperature prediction and directly affects the capacity of defect detection of this method. In this paper, we proposed an inverse method to characterize concrete hydration model parameters using evolutionary optimization. With this approach, the finite element analysis is integrated into the differential evolution algorithm to generate approximate solutions that match a controlled dataset instead of approximating hydration parameters with limited prior knowledge as currently used in practice. The method overcomes the uncertainties of concrete mixes and material thermal properties. The field case study further verifies that the proposed inverse approach using DE algorithms can be used effectively in thermal integrity testing. 


\section{ACKNOWLEDGEMENT}

This work was performed in the framework of ITN-FINESSE, funded by the European Union's Horizon 2020 research and innovation program under the Marie SkłodowskaCurie Action grant agreement $\mathrm{n}^{\circ} 722509$.

\section{REFERENCES}

De Schutter, G. \& Taerwe, L. (1995). General hydration model for Portland cement and blast furnace slag cement. Cement and Concrete Research, 25(3), 593-604.

De Schutter, G. \& Taerwe, L. (1996). Degree of hydration-based description of mechanical properties of early age concrete. Materials and structures, 29(6), 335.

Jena, P. K., Thatoi, D. N. \& Parhi, D. R. (2013). Differential evolution: an inverse approach for crack detection. Advances in Acoustics and Vibration, 2013.

Leung, Y. F., Klar, A., Soga, K. \& Hoult, N. A. (2017). Superstructure-foundation interaction in multi-objective pile group optimization considering settlement response. Canadian Geotechnical Journal, 54(10), 1408-1420.

Liu, G. \& Mao, Z. (2017). Structural damage diagnosis with uncertainties quantified using interval analysis. Structural Control and Health Monitoring, 24(10), e1989.

Malhotra, V. M. \& Carino, N. J. (2003). Handbook on non-destructive testing of concrete. CRC press.

Riding, K. A., Poole, J. L., Schindler, A. K., Juenger, M. C. \& Folliard, K. J. (2006). Evaluation of temperature prediction methods for mass concrete members. ACI Materials Journal, 103(5), 357-365.

Rui, Y., Kechavarzi, C., O’Leary, F., Barker, C., Nicholson, D. \& Soga, K. (2017). Integrity testing of pile cover using distributed fibre optic sensing. Sensors, 17(12), 2949.

Schindler, A. K. (2004). Effect of temperature on hydration of cementitious materials. Materials Journal, 101(1), 72-81.

Schindler, A. K. \& Folliard, K. J. (2005). Heat of hydration models for cementitious materials. ACI Materials Journal, 102(1), 24.

Storn, R. \& Price, K. (1997). Differential evolution-a simple and efficient heuristic for global optimization over continuous spaces. Journal of global optimization, 11(4), 341-359.

Sun, Q. \& Elshafie, M. Z. E. B. (2019). A new thermal integrity method for pile anomaly detection. The 12th International Workshop on Structural Health Monitoring. September 10-12, 2019, Stanford, California, the USA.

Sun, Q., Elshafie, M. Z. E. B., Backer, C., Fisher, A. \& Rui, Y. (2020). Thermal integrity testing of cast in-situ piles using a new interpretation approach. Structural health monitoring, journal in progress.

Uchida, S., Xie, X. G. \& Leung, Y. F. (2016). Role of critical state framework in understanding geomechanical behavior of methane hydrate-bearing sediments. Journal of geophysical research: solid earth, 121(8), 5580-5595. 